ionization zone, but magnetic fields play an important role in mode selection. Studies of these stars may help us to understand the nature of Ap stars in general.

In $\delta$. Scuti star models, unstable modes cover a wide range of frequencies around the fundamental radial mode frequency. The instability extends to high spherical harmonic degrees. The driving effect is essentially the same as in the classic pulsating variables. Unstable modes include low order $g$-modes which probe very well the $\mu$-gradient surrounding the convective core. Treatment of such modes remains subject of controversy.

The origin of pulsation in $\beta$ Cephei stars has been an outstanding puzzle for stellar stability theory. The improved Iglesias \& Rogers (1992) data lead to a fully satisfactory solution in terms of an opacity mechanism acting in the metal bump zone. The unstable modes are similar to those in $\delta$ Scuti stars though the instability does not extend to p-modes of higher order than $p_{1}$ or $p_{2}$. Studies of $\beta$ Cephei star pulsation have the potential for probing the interiors of massive stars.

The SPB stars are a newly defined group of pulsating stars (Waelkens 1992) containing Main Sequence objects in the spectral type range B3 - B8 which are variable with periods from 0.5 to 4 days. The observational evidence for $\mathrm{g}$-mode excitation was quite convincing. Subsequently it has been shown that the same mechanism that causes pulsations in $\beta$ Cephei stars in less massive objects drives high-order g-modes with periods consistent with those determined in SPB stars. These modes are excellent probes of the whole radiative interior, however the much longer period presents a very different challenge for observers than in the case of the other types of pulsating Main Sequence stars.

\title{
ATMOSPHERES AND WINDS OF HOT STARS - IMPACTS OF NEW OPACITY CALCULATIONS AND CONTINUING NEEDS
}

\author{
KEITH BUTLER \\ Universitäts - Sternwarte München, Scheinerstr. 1, 81679 München, Germany
}

In this paper I review some recent advances in the use of large amounts of atomic data in the modelling of atmospheres and winds of hot stars. The review is highly selective but representative of current developments. A more general overview is to be found in Kudritzki and Hummer (1990) although the field is changing so rapidly that much has happened since then. The paper breaks down into three parts: work on line formation, in which the atmospheric structure is known and held fixed, is described first, then follows a description of the inclusion of line opacities in non-LTE in the atmosphere problem itself, and finally recent developments in the theory of radiatively driven stellar winds are summarized. Here special emphasis is given to a novel distance determination method based 
entirely on spectroscopic quantities. I close with a brief shopping list.

In a series of papers, Becker and Butler $(1992,1994 a, b, c)$ have investigated iron and nickel spectra in sub-dwarfs using the complete linearization method of Auer and Heasley (1976). The method scales linearly with the number of frequency points so they were able to use well over 10000 frequencies to adequately describe the line opacities. Several thousand lines were treated explicitly and the resultant computed spectra gave execellent fits to observed Hubble spectra in the wavelength ranges dominated by the ions concerned.

The different ionization stages gave consistent results for the iron and nickel abundances but only after line-blocking from millions of spectral lines in the far UV had been included. This was done using the Kurucz (1988) line lists coupled with line grouping as suggested by Anderson (1989) and described briefly in the next section.

The line-blanketed atmospheres of Kurucz (1991) are the best available up to about $30000 \mathrm{~K}$, where non-LTE effects start to become important. Non-LTE line-blanketed atmospheres have become feasible because the computational requirements of the accelerated lambda iteration (ALI) method (Werner and Husfeld, 1985) also scale linearly with the number of frequency points. On the other hand, Anderson (1989) suggested grouping energetically adjacent atomic levels together to form pseudo-levels on the basis that although they might, as a group, be in non-LTE, they should be in LTE with respect to one another due to the large number of collisions between them. This greatly reduces the number of levels to be considered but instead gives rise to highly complicated pseudo lineprofiles. Grigsby et al (1992), who did not use ALI, constructed the first grid of lineblanketed non-LTE models by using a variation on the Opacity Distribution Function concept to group line opacities into blocks thereby reducing the number of frequency points required. Dreizler and Werner (1993) on the other hand were able to sample the opacity as they used ALI in their models.

Pauldrach et al (1994) have presented a new set of radiatively-driven wind models which include not only greatly improved opacities but also line blocking and the effect of shocks on the EUV spectrum. The improved opacities increased the mass loss rates by a factor of two compared to earlier calculations while the shocks and line blocking allow various ionization stages to give consistent results. The new models reproduce the observed UV spectra of two massive O stars, $\zeta$ Puppis in our Galaxy and Melnick 42 in the LMC extremely well. The latter is found to be much hotter than a previous analysis had suggested as here the stellar wind is found to be much more dense. Abundances for a variety of elements were also derived from the UV spectrum alone. Based on such models the theory shows that there is a very tight relationship between the stellar luminosity on the one hand and the quantity $\mathrm{Mv}_{\infty} \mathrm{R}^{0.5}$ on the other. Since the radius, mass-loss rate and end velocity can be obtained from the observed spectra the luminosity and hence the distance may be thus obtained. The observations (Lennon et al, 1995) demonstrate that the relation holds over a wide range of spectral type and also in other galaxies. It is, however, affected by metallicities thus making it imperative that these also be available if the method is to be effective. 
Work on A supergiants is now underway opening up the prospect of spectroscopic distance determinations over cosmologically significant ranges with the coming generation of $8 \mathrm{~m}$ ground-based telescopes.

In constructing the model atoms and calculating the atomic data needed, it soon becomes apparent that one of the most serious deficiencies is the small number of energy levels and wavelengths available for many of the iron group ions. These are important for spectral synthesis. Moderately accurate collisional data, partial photoionization cross sections and many more line broadening data would also be most welcome.

Anderson, L.A., 1989, ApJ 339, 558

Auer, L.H., Heasley, J.N., 1976, ApJ 205, 165

Becker, S.R., Butler, K., 1992, A\&A 265, 647

Becker, S.R., Butler, K., 1994a, A\&A in press

Becker, S.R., Butler, K., 1994b, A\&A in press

Becker, S.R., Butler, K., 1994c, A\&A submitted

Dreizler, S. and Werner, K., 1993, A\&A 278, 199

Grigsby, J.A., Morrison, N.D. and Anderson, L.S., 1992, ApJS 78, 205

Kudritzki, R.P. and Hummer, D.G., 1990, Ann, Rev, Astron. and Astrophys. 28, 030

Kudritzki, R.P., Lennon, D.H. and Puls, J., 1995, in "Science with the VLT", ESO Conf., in press

Kurucz, R.L., 1988, Transactions of the Internal Astronomical Union, M. McNally, ed., Dordrecht: Kluwer, XXB, 168

Kurucz, R.L., 1991, in Stellar Atmospheres: Beyond Classical Models, NATO

ASI series C, Vol 341, eds L. Crivalleri, I. Hubeny and D.G. Hummer, Kluwer, Dordrecht, p.441 Pauldrach, A.W.A., Kudritzki, R.P., Puls, J., Butler, K. and Hunsinger, 1994, A\&A 283, 525

Werner, K. and Husfeld, D., 1985, A\&A 148, 417

\title{
WHAT NEBULAR OBSERVATIONS AND PHOTOIONIZATION MODELS CAN TELL US ABOUT ATMOSPHERES OF HOT STARS
}

\author{
ROBERT H. RUBIN ${ }^{1,2}$, DIETMAR KUNZE ${ }^{3}$, AND TETSUO YAMAMOTO
}

${ }^{\prime}$ NASA-Ames Research Center, MS 245-6, Moffett Field, CA 94035-1000

${ }^{2}$ Orion Enterprises, 2135 Woodleaf Way, Mountain View, CA 94040

${ }^{3}$ Max Planck Institut, für Astronomy, D-81679 München, Germany

'Institut fur Astronomie und Astrophysik der Universität München, Scheinerstr. 1, 81679 München, Germany

A major purpose of this paper is to emphasize to the stellar atmosphere community 